\title{
X-Ray Emission From Betatron Motion In A Plasma Wiggler
}

\author{
Shuoqin Wang ${ }^{1}$, C. E. Clayton ${ }^{1}$, B. E. Blue ${ }^{1}$, E. S. $\operatorname{Dodd}^{1}$, K. A. Marsh ${ }^{1}$, W. B. Mori ${ }^{1}$, C. \\ Joshi $^{1}$, S. Lee ${ }^{2}$, P. Muggli ${ }^{2}$, T. Katsouleas ${ }^{2}$, F. J. Decker ${ }^{3}$, M. J. Hogan ${ }^{3}$, R. H. Iverson ${ }^{3}$, \\ P. Raimondi ${ }^{3}$, D. Walz ${ }^{3}$, R. Siemann ${ }^{3}$, R. Assmann ${ }^{4}$ \\ ${ }^{1}$ University of California, Los Angeles, CA $90095,{ }^{2}$ University of Southern California, Los \\ Angeles, CA 90089, ${ }^{3}$ Stanford Linear Accelerator Center, Stanford, CA 94309, ${ }^{4}$ CERN, \\ Switzerland
}

\begin{abstract}
The successful utilization of an ion channel in a plasma to wiggle a $28.5 \mathrm{GeV}$ electron beam to obtain broad band X-ray radiation is reported. The ion channel is induced by the electron bunch as it propagates through an underdense 1.4 meter long lithium plasma. The quadratic density dependence of the spontaneously emitted betatron $\mathrm{X}$-ray radiation and the divergence angle of $(1 \sim 3) \times 10^{-4}$ radian of the forward emitted $\mathrm{X}$-rays as a consequence of betatron motion in the ion channel are in good agreement with theory. The absolute photon yield and the peak spectral brightness at $14.2 \mathrm{KeV}$ photon energy are estimated.
\end{abstract}

52.59.Ye, 52.59.Rz, 52.59.Px

Typeset using REVTEX

1

Work supported in part by the Department of Energy contract DE-AC03-76SF00515. 
Synchrotron light sources are used for basic and applied research in physical, chemical and biological sciences, and in engineering [1]. These use magnetic undulators and wigglers to generate high brightness photon beams in the $\mathrm{X}$-ray region using relativistic electron beams. In this paper we show that an ion channel induced by an electron beam as it propagates through a plasma can wiggle the beam electrons to generate $\mathrm{X}$-ray radiation. Because a dense column of ions can provide an effective wiggler strength that can be much greater than that provided by a conventional magnet, such plasma wigglers /undulators [2] [3] [4] could impact future generation light sources.

It is well known that an electron beam injected into an ion column undergoes betatron oscillations of its transverse envelope or beam size [5]. An individual electron within this beam executes simple harmonic motion about the axis of the ion channel with betatron frequency $\omega_{\beta}=k_{\beta} c=\omega_{p} / \sqrt{2 \gamma_{b}}$ where $\omega_{p}=\sqrt{4 \pi n e^{2} / m}$ is the plasma frequency, $n$ is the plasma density, and $\gamma_{b}$ is the relativistic Lorentz factor of the beam. The betatron motion of a relativistic electron with an initial displacement $r_{0}$ from the axis of the ion channel is described by $\vec{r}=\overrightarrow{r_{0}} \cos \phi$, leading to $\vec{\beta}_{r}=-\overrightarrow{r_{0}} k_{\beta} \sin \phi$, and $\dot{\vec{\beta}}_{r}=-\overrightarrow{r_{0}} k_{\beta} \omega_{\beta} \cos \phi$ with $\dot{\phi}=\omega_{\beta}$. The total radiation power as a consequence of this motion is given by [6] $P(t)=\frac{2 e^{2} \gamma_{b}^{2}}{3 m^{2} c^{3}}\left[\dot{\vec{p}}^{2}-m^{2} c^{2} \dot{\gamma}_{b}^{2}\right] \approx \frac{2}{3} c e^{2} \gamma_{b}^{4} r^{2} k_{\beta}^{4} \cos ^{2} \phi$ where $\vec{p}_{r}=\gamma_{b} m c \vec{\beta}_{r}$. Therefore the total power is proportional to $\omega_{\beta}^{4}$ which scales as the square of the plasma density. The spectrum of this betatron radiation has resonance frequencies at $\omega_{r}=2 m_{h} \gamma_{b}^{2} \omega_{\beta} /\left(1+K^{2} / 2+\left(\gamma_{b} \Omega\right)^{2}\right)$ where $m_{h}=1,2,3 \cdots$ is the harmonic number [7]. The wiggler strength $K$ is given by $K=\gamma_{b} \omega_{\beta} r_{0} / c$, and $\Omega<<1$ is the observation angle measured from the axis. For a beam with a transverse size $\sigma_{r}$, each electron has a different $r_{0}$ and radiates a different $\omega_{r}$ and the spectrum of radiation therefore tends to be broad. If $K>>1$, high harmonic radiation dominates the spectrum and a broadband spectrum is generated as from a conventional wiggler. This broadband spectrum is characterized by the critical frequency [6] given by $\omega_{c}=3 \gamma_{b}^{3} c r_{0} k_{\beta}^{2} / 2$. The full width at half-maximum (FWHM) beam divergence angle is $\theta \sim K / \gamma_{b}$ which can be extremely narrow for an ultra-relativistic beam. Although microwave radiation has been observed in this underdense or ion-focussed regime [8], no measurements 
of X-rays have been reported to our knowledge.

The experiment was carried out at the Stanford Linear Accelerator Center (SLAC) using the Final Focus Test Beam (FFTB). The 28.5 GeV SLAC beam containing typically $1.8 \times$ $10^{10}$ electrons per bunch (with $\sigma_{r}=40 \mu \mathrm{m}$ and a longitudinal bunch length $\sigma_{z}=0.7 \mathrm{~mm}$ ) was focussed near the entrance of a lithium plasma of length $L_{p}=1.4 \mathrm{~m}$ produced by single photon ionization of a lithium-vapor column by an ArF laser [9]. Therefore the plasma density, in the optically thin limit, is simply proportional to the total laser energy absorbed by the plasma. The plasma density was thus varied by varying the laser energy. The plasma density deduced from the UV absorption measurements was calibrated against a more direct measurement of plasma density using $\mathrm{CO}_{2}$ interferometry in an offline experiment [10]. The typical plasma diameter was $2 \mathrm{~mm}$. A schematic of the experimental set-up is shown (not to scale) in Fig. 1. The electron beam had a small and approximately linear "head-to-tail" tilt when it entered the plasma [11]. Over the range of plasma densities used in this experiment, the peak beam density was larger than the plasma density. In this so-called underdense plasma regime, the head of the beam expels the plasma electrons creating an ion channel. This ion channel in turn exerts a transversely-linear and longitudinally-uniform focusing gradient on the main body of the electron beam. At the highest density of $1.7 \times 10^{14} \mathrm{~cm}^{-3}$, the magnitude of this force, about $50 \mathrm{MG} / \mathrm{m}$, is enough to cause the beam to focus from the initial $40 \mu \mathrm{m}$ to $<5 \mu \mathrm{m}$ after propagating only $\sim L_{p} / 6$ inside the plasma. The electrons in the beam overshoot this focus (cross the axis) and the beam radius returns to the initial size after propagating $\sim L_{p} / 3$. Thus the beam undergoes about three envelope oscillations $\left(k_{\beta} L_{p} \sim 3 \pi\right)$ within the full length of the plasma, whereas the individual electrons execute 1.5 betatron oscillations, crossing the axis at integer $\pi$ values of $k_{\beta} L_{p}$.

These betatron oscillations were simultaneously measured in two ways. The first measurement was of the beam size 1 meter downstream of the plasma as observed from optical transition radiation (OTR) [12]. This showed that as the plasma density was raised slowly beginning from $1 \times 10^{13} \mathrm{~cm}^{-3}$, the radius of the beam on the OTR foil would at first increase (over-focused) but then drop back down to approximately the plasma-off size when 
$k_{\beta}(n) L_{p} \sim \pi$. This envelope oscillation continued [13] as $n$ was further increased, giving a beam-size minimum at $k_{\beta} L_{p} \sim 2 \pi$ and another at $k_{\beta} L_{p} \sim 3 \pi$ when $n \sim 1.7 \times 10^{14} \mathrm{~cm}^{-3}$. These values of $n$ where $k_{\beta} L_{p} \sim m \pi(m=1,2,3)$ are called "transparency points" since the beam spot size coming out is similar to the beam size at the same location without the plasma. In the second, concurrent measurement shown in Fig. 2(a), the center of mass (centroid) of the beam was measured (using a beam position monitor placed $2.6 \mathrm{~m}$ from the plasma exit) as $n$ was increased. Due to the initial tilt on the beam, the centroid of the bunch had a small transverse offset from the axis of the ion channel, since it is the head of the bunch that defines this axis. Consequently, the motion of the centroid behaves much like that of a single electron which starts at some offset from the ion channel axis, i.e., the centroid crosses the axis at $k_{\beta} L_{p} \sim m \pi(m=1,2,3)$. This is indeed what is observed in Fig. 2(a) where the centroid displacement returns to its initial offset $(\sim 0)$ at the same values of $n$ where the beam size had a minimum [13] (the arrows labeled 1,2, and 3 in Fig. 2(a)). Both the envelope and centroid oscillations agree reasonably well with the theoretical predictions (solid line in Fig. 2(a)) based on the beam envelope equation for beam propagation in a uniform ion channel [13] [14] up to a density of about $1.7 \times 10^{14} \mathrm{~cm}^{-3}$. In fact, the absolute plasma density shown in Figs 2(a) and (b) derived from this theoretical fit is close to that inferred from absolute absorption of the ionizing laser beam.

The electron beam exiting the plasma was bent away using a $5.2 \mathrm{~m}$ long dipole magnet located three meters from the exit of the plasma. The X-rays emitted by the undulating electrons were recorded $40 \mathrm{~m}$ downstream of the plasma after traversing the $25 \mu \mathrm{m}$ thick titanium OTR foil at $45^{\circ}$ with respect to the beam axis and two $75 \mu \mathrm{m}$ thick beryllium windows. The X-rays were reflected by a $1 \mathrm{~mm}$ thick silicon (111) crystal and detected using two $1 \mathrm{~mm}$ thick silicon surface barrier detectors (SBD). One of the SBDs (D2) could be placed to precisely receive the Bragg-reflected X-rays, while the other (D1) was placed in the same plane but at a mismatched angle to receive Thomson-scattered photons from the crystal. Both detectors were well shielded using lead to decrease X-ray noise that is incident from directions other than the photon beam line. The signal on the SBDs is due to photons 
in the $5 \sim 30 \mathrm{KeV}$ energy range because photons with energies less than $5 \mathrm{KeV}$ are mainly attenuated by the Ti foil while those with energies greater than $30 \mathrm{KeV}$ transmitted through the detector.

Figure 2(b) shows the integrated $\mathrm{X}$-ray power (arbitrary units) in the energy range $5 \mathrm{KeV}$ to $30 \mathrm{KeV}$ (red dots) as a function of plasma density using detector D1 that received Thomson-scattered X-rays. Also shown are the relative values (blue triangles) of the theoretically expected X-ray energy yield by numerically integrating the expression for the Lienard-Wiechert potential [6] [15] over all solid angles within the $5 \sim 30 \mathrm{KeV}$ photon energy range at the transparency points, and taking into account corrections for the transmission function of the metal foils, and the frequency response of the atomic scattering factor of silicon atoms. The details of this calculation are presented in [16]. At low densities $\left(n<1.0 \times 10^{14} \mathrm{~cm}^{-3}\right)$ the shot-to-shot variation in the $\mathrm{X}$-ray energy is relatively small compared to that at higher densities. The fluctuations at higher densities are caused by the $n^{2}$-dependence of the X-ray power and the fact that the actual plasma density can fluctuate $\pm 15 \%$ about the deduced plasma density due to experimental limitations such as spatial fluctuations in the ionizing UV profile. The agreement between theory and experiment is good. Also shown is a curve representing the expected quadratic dependence of the X-ray energy on plasma density. Even though both the measurement and the numerical integration are over the $5 \sim 30 \mathrm{KeV}$ photon-energy range, they match well with the expression for total energy-yield over all frequencies which scales as $n^{2}$.

We have measured the absolute value of photon numbers around the $m=3$ transparency point $\left(n=1.5 \sim 1.8 \times 10^{14} \mathrm{~cm}^{-3}\right.$ ) using the Bragg scattering technique. As can be seen from the inset to Fig. 3, as the Si crystal is rotated the signal received by detector D2 shows an enhanced peak at $\hbar \omega \sim 14.2 \mathrm{KeV}$ when the exact Bragg condition is satisfied at $\theta_{S i} \sim 8^{\circ}$. The expected bandwidth of the Bragg signal at the $m=3$ transparency point is determined by the range of incident angles within the X-ray beam. As stated before, this range is given by $\Delta \theta \sim K / \gamma$ which for $m=3, \sigma_{r}=40 \mu m$, and $K=16.8$ is $\Delta \theta \sim 0.29$ mrad. From $\Delta \omega / \omega=-\Delta \lambda / \lambda=-\Delta \theta / \tan \theta$ we have $\Delta \omega / \omega=0.002$. Detector D2 subtends an angle off 
the Si crystal of $\sim 10 \mathrm{mrad}>\Delta \theta$ and thus easily captures this bandwidth.

We acquired two sets of data while varying the plasma density by $\sim \pm 10 \%$ around the third transparency point. In the first set of data both D1 and D2 were not at the Bragg angle and therefore received radiation in the same broadband photon energy range. The signals on the two detectors for this data set are plotted against each other as triangles in Fig. 3, essentially showing the relative sensitivities of the two detector systems. The crystal was then rotated to $\theta_{S i} \sim 8^{\circ}$ such that D2 received, in addition to the broadband radiation, Bragg scattered photons at $14.2 \mathrm{KeV}$ while D1 still received only the broadband radiation. A plot of the signals on D1 vs. D2 (rectangles in Fig. 3)shows that for the same range of signals on D1, D2 received $\sim 2.1 \times$ more signal when at Bragg angle. We attribute this $\sim 250 \mathrm{mV}$ average additional signal to the $14.2 \mathrm{KeV}$ Bragg-scattered photons. After taking into account the detector circuit response, the photon-to-electron conversion efficiency of the SBD and the transmission loss of the system, we estimate the number of $14.2 \mathrm{KeV}$ photons to be $6 \times 10^{5}$. This number is to be taken as a lower bound as we have assumed a Bragg reflectivity of unity.

We now compare the measured number of photons at $14.2 \mathrm{KeV}$ with a theoretical estimate. The number of photons in the above mentioned energy bin can be estimated by approximating the radiation spectrum of our plasma wiggler, $W$, with that of a bending magnet, B. We thus have $\left[\frac{d N_{p h}}{d \psi}\right]_{W}=m\left[\frac{d N_{p h}}{d \psi}\right]_{B}=m \cdot C_{\psi} \cdot I \cdot E \cdot \frac{\Delta \omega}{\omega} \cdot S\left(\omega / \omega_{c}\right)$, where $C_{\psi}=3.967 \times 10^{16}$ photons $/(\mathrm{sec} \cdot \operatorname{mrad} \cdot A m p \cdot G e V)$, and $S\left(\omega / \omega_{c}\right)=\frac{9 \sqrt{3} \omega}{8 \pi \omega_{c}} \int_{\frac{\omega}{\omega_{c}}}^{\infty} K_{5 / 3}(x) d x$ is the so-called universal function [17]. In the above expression, $I$ is the average beam current within an electron bunch, $E$ is the beam electron energy, $m$ is the integral number of the betatron periods $(m=3), \psi$ is the sweep angle $(\sim K / \gamma), \omega$ satisfies $\hbar \omega \sim 14.2 K e V$, and $\Delta \omega / \omega$ is is the normalized bandwidth $(=0.002)$. Based on the above numbers, we obtain $N_{p h} \sim 3.0 \times 10^{6}$ at $14.2 \pm 0.014 \mathrm{KeV}$. This estimate is about five times larger than the measured number.

Using the $14.2 \mathrm{KeV}$ photon yield deduced at $m=3$ and a radiation angle of $\sim K / \gamma_{b}$, we can estimate the photon integrated-intensity [15] to be $4.5 \times 10^{17}$ photons $/\left(\mathrm{sec} \cdot \mathrm{mrad}^{2}\right.$. 
$0.1 \%$ bandwidth). The effective source size $S$ of the $14.2 \mathrm{KeV}$ radiation can be approximated as $S=\pi\left(\sigma_{r}^{2}+\frac{1}{4}\left(\left(\epsilon / \sigma_{r}\right)^{2}+\left(0.6 \mathrm{~K} / \gamma_{b}\right)^{2} L_{p}^{2}\right) \sim 0.06 \mathrm{~mm}^{2}\right.$ where $\epsilon$ is the geometrical emittance of the beam. We therefore estimate the peak spectral brightness to be about $7 \times 10^{18}$ photons $/\left(\mathrm{sec} \cdot \mathrm{mrad}^{2} \cdot \mathrm{mm}^{2} \cdot 0.1 \%\right.$ bandwidth $)$.

Figure 4 shows a processed image of the fluorescence produced by the X-rays from betatron motion in the plasma ion column at the first transparency point $(m=1)$. To obtain this image, a plasma-off image was subtracted from a plasma-on image. With the plasma off, the $\mathrm{X}$-rays producing the fluorescence are generated by synchrotron radiation due to all the multipole magnets (not shown in Fig. 1) upstream of the dipole bend magnet, bremsstrahlung from the metallic foils, and synchrotron radiation due to the dipole bend magnet used to separate the photons from the electrons. With the plasma on, the fluorescence is generated by $\mathrm{X}$-rays from all the above mentioned sources and $\mathrm{X}$-rays due to betatron motion in the plasma. Because of the small initial tilt on the beam, the beam exits the plasma with a small deflection angle. This makes perfect subtraction of the contribution of the downstream dipole bend radiation impossible. Thus Fig. 4 shows a round spot corresponding to the "betatron" X-rays and a vertical tail that is left over from subtraction of this misaligned dipole bend radiation. The FWHM of the plasma "betatron X-ray" fluorescence image is approximately $4 \mathrm{~mm}$ which gives a full-beam divergence angle of $10^{-4}$ radian. The theoretical estimate for the angle is roughly $K / \gamma_{b} \sim 0.9 \times 10^{-4}$ for the present condition. Thus the measured angle is in good agreement with the theory, particularly since the fluorescent image is dominated by lower energy X-ray photons. These results are the first observation of plasma betatron radiation in the X-ray region to our knowledge.

We have shown that an ion channel induced by the beam can wiggle beam electrons to produce X-radiation. Such a plasma wiggler comprising of only a plasma cell offers simplicity of construction, flexibility in undulator wavelength, and potential savings of cost over its magnetic wiggler counterpart in future generation of light sources. The electron beam hosing instability could ultimately limit the number of periods of oscillation by causing transverse break-up of the beam in such a device [18]. 
We are grateful to W.H. Tompkins of SSRL for his support with the X-ray diagnostics and E.Esarey of LBNL for valuable comments. We thank D.Whittum and S.Rokni of SLAC, and W.P.Leemans, P. Catravas, and S. Chattopadhyay of LBNL for their help.This work was supported by DOE grants DE-AC03-76SF00515, DE-AC-03-76SF0098, and DE-FG0398-DP-0021 and NSF grant ECS9617089. 


\section{REFERENCES}

[1] H. Winick, Scientific American, 11, 88, (1987).

[2] C.Joshi, et al., IEEE Journal of Quantum Electronics 23, 1573 (1987).

[3] R. Fedele, et al., Physica Scripta 30, 192 (1990).

[4] R. L. Williams, et al., IEEE Trans. Plasma Sci.,21, 166, (1993).

[5] D. H. Whittum, et al., Phys. Fluids 4, 730, (1992).

[6] J. D. Jackson, Classical Electrodynamics, 662, J. Wiley and Sons, N. Y. (1975).

[7] E. Esarey et al., AIP Proceedings, 569, 473, (2001).

[8] D. H. Whittum, et al., IEEE Trans. Plasma Sci., 21, 136, (1993).

[9] M. Hogan, et al., Phys. Plasmas 7, 2241, (2000).

[10] P. Muggli, et al., IEEE Trans. Plasma Sci., 27, 791, (1999).

[11] B. Blue, et al., Proceedings of the 2001 Particle Accelerator Conference (2001).

[12] D.W.Rule, et al., Nucl. Instrum. Methods Phys. Res., Sect. B 24/25, 901, (1987).

[13] C. Clayton, et al. submitted to PRL.

[14] J. D. Lawson, The physics of charged particle beams (second edition), Oxford U. P., London, 161, (1988).

[15] K. J. Kim, AIP Con. Proceedings184, 565, (1989).

[16] S.Wang, et al., Proceedings of the 2001 Particle Accelerator Conference (2001).

[17] H. Wiedemann, Particle Accelerator Physics II, Sprnger, 266, (1995).

[18] A. A. Geraci and D. H. Whittum, Phys. Plasmas, 7, 3431, (2000). 


\section{FIGURES}

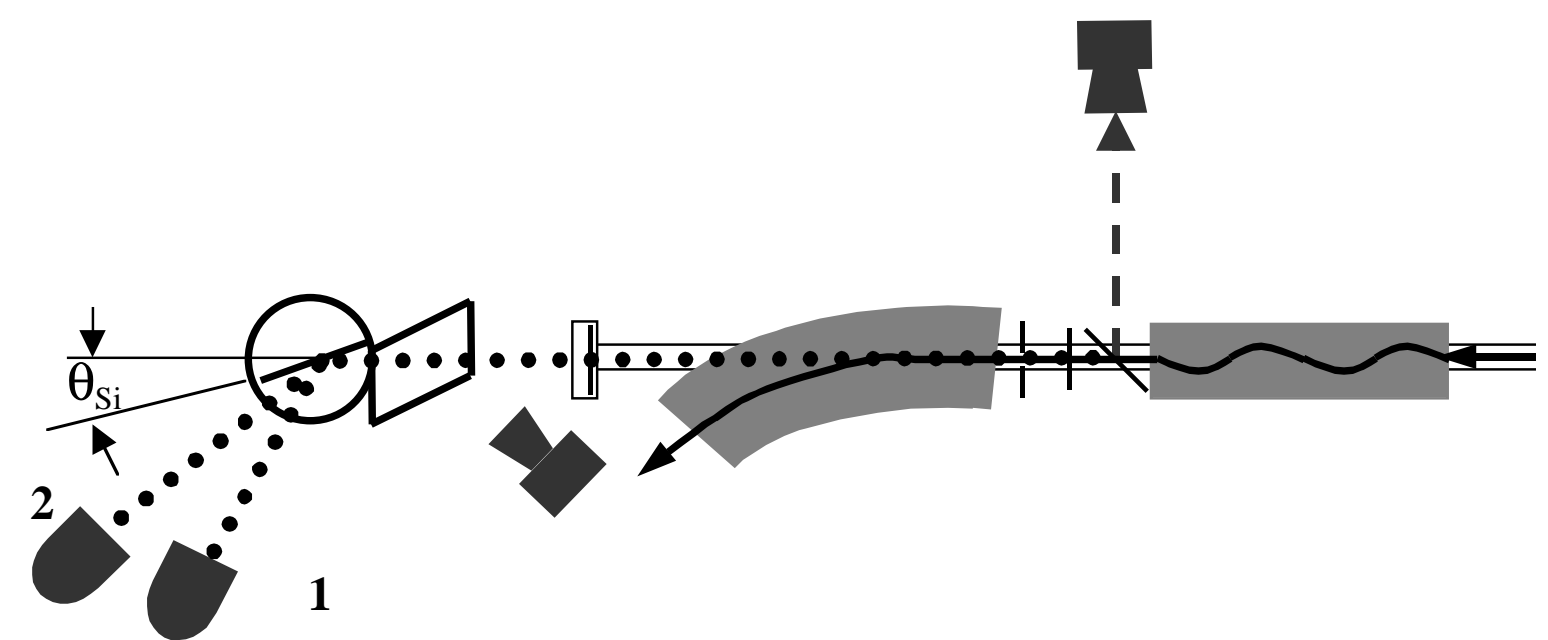

FIG. 1. Schematic of the experimental set-up. A bend magnet separates the electrons (solid red line) from the photons (dashed blue line) after the plasma wiggler. Key: A-lithium plasma source; B-OTR Ti foil; C-CCD cameras; D1-SBD receiving Thomson scattered X-rays; D2-SBD receiving Bragg scattered X-rays; E-Beryllium vacuum windows; F-bend magnet; G-photon beam line; H-fluorescent screen, I-silicon (111) crystal on a rotation stage at angle $\theta_{S i}$, J-beam position monitor. 


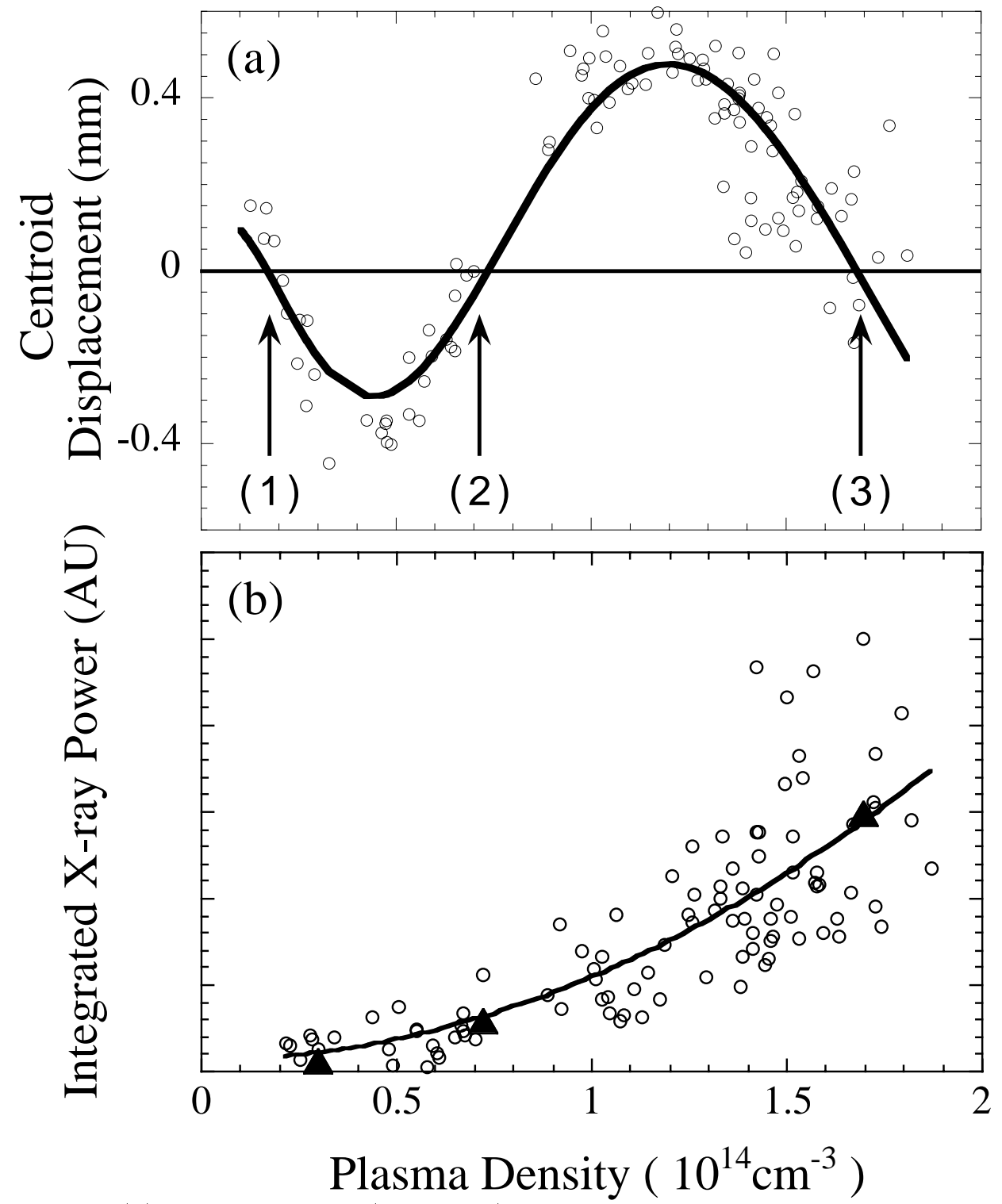

FIG. 2. (a) The predicted (solid line) and measured displacement of the beam centroid as a function of plasma density. (b) The estimated (triangles) and the measured (dots) X-ray energy in the $5 \sim 30 \mathrm{KeV}$ range as a function of plasma density. The solid line is the quadratic fit to the experimental data. 


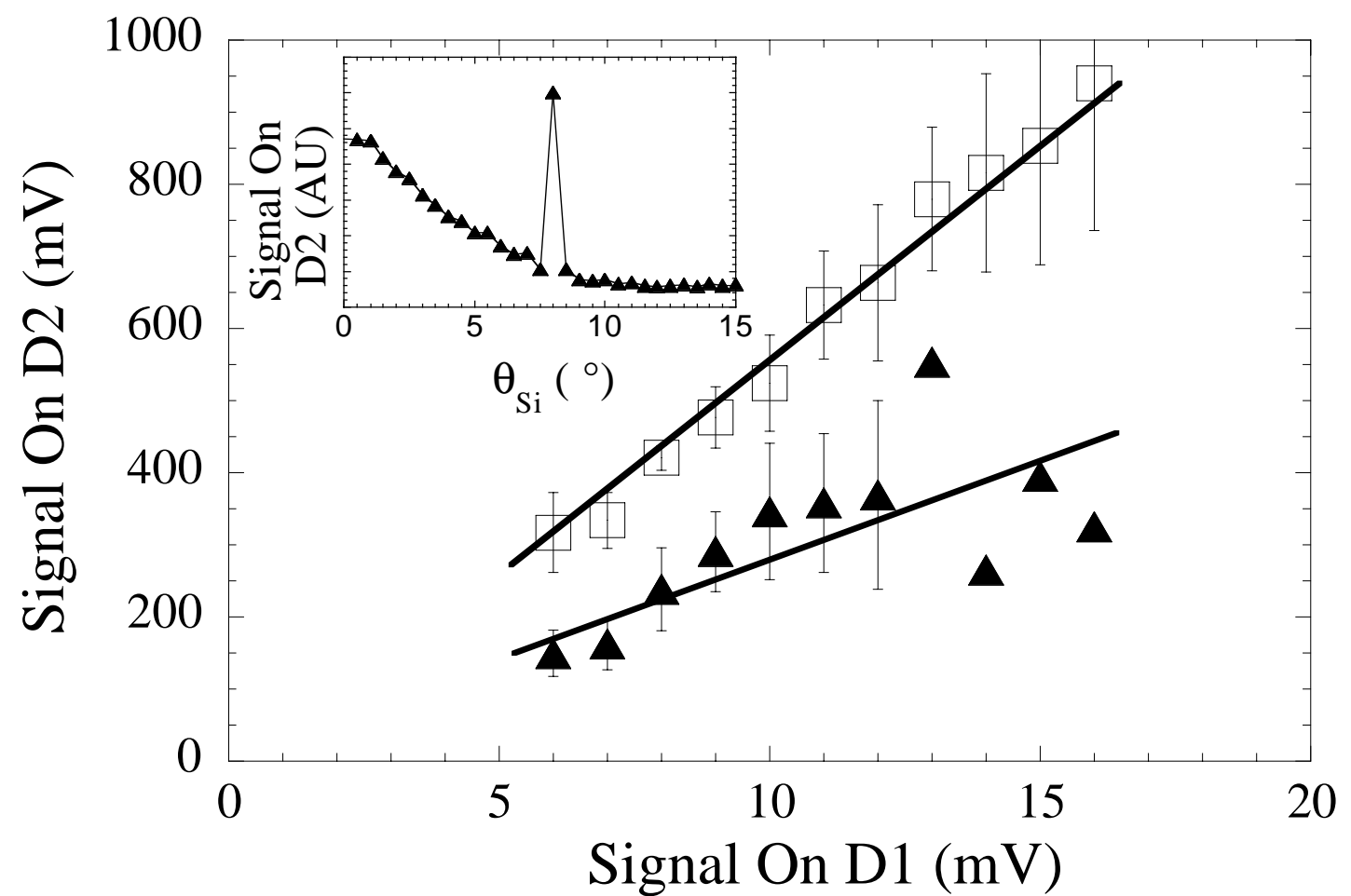

FIG. 3. The correlation of signals on detector D2 vs. detector D1. The inset shows clear Bragg peak at $8^{\circ}$ on D2 as $\theta_{S i}$ is varied (curve taken with plasma off and without the Ti OTR foil in the beam line). 


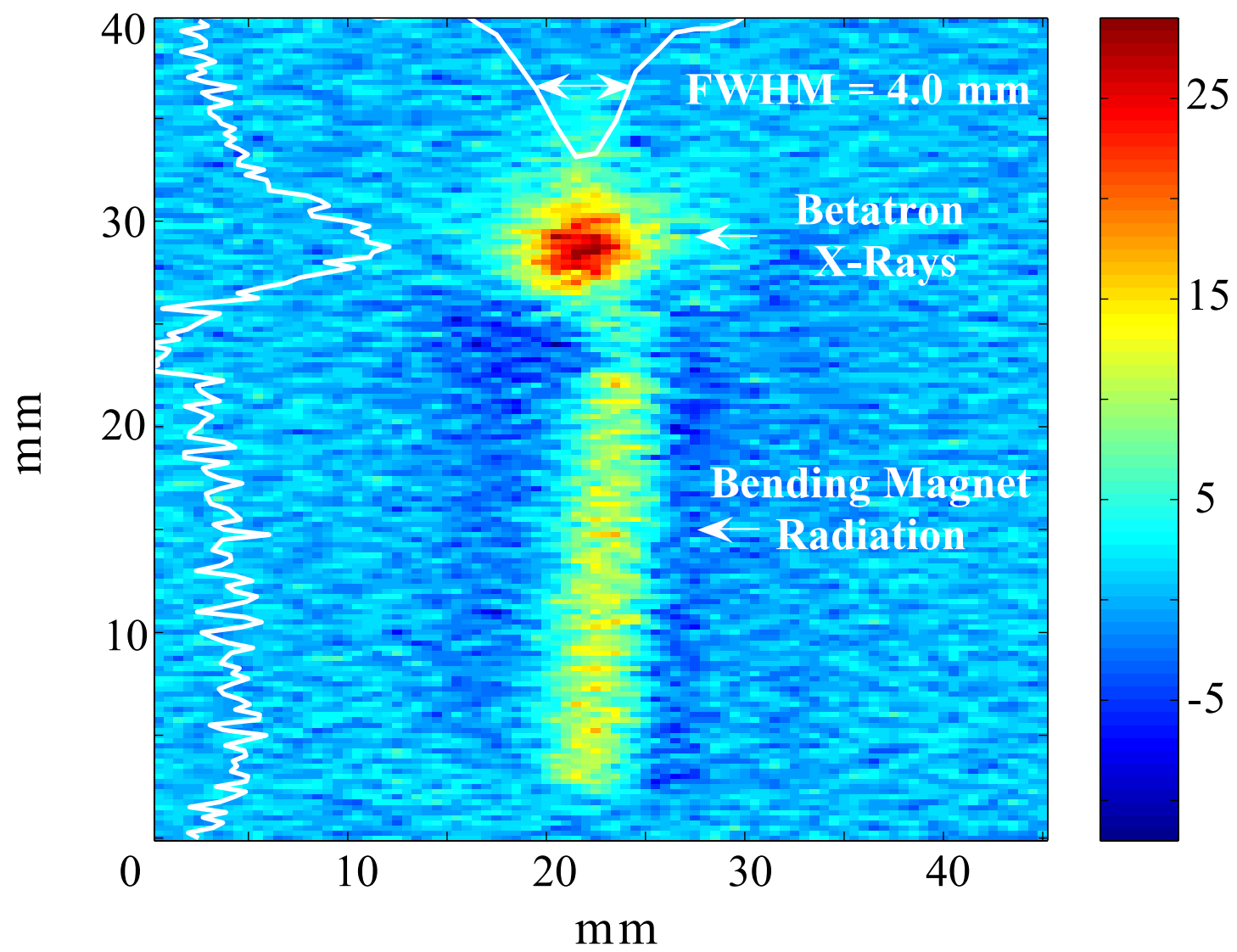

FIG. 4. Processed image produced on the fluorescent screen as recorded by the CCD camera showing the "betatron" X-rays produced by the plasma (circle at the top) and a vertical stripe of remnant synchrotron radiation produced by the bend dipole magnet. 